\section{Michigan Technological 188 5 University}

Michigan Technological University Digital Commons @ Michigan Tech

\title{
Mechanical Properties of Components Fabricated with Open- Source 3-D Printers Under Realistic Environmental Conditions
}

\author{
B. M. Tymrak \\ Michigan Technological University \\ M Kreiger \\ Michigan Technological University \\ Joshua M. Pearce \\ Michigan Technological University
}

Follow this and additional works at: https://digitalcommons.mtu.edu/materials_fp

Part of the Materials Science and Engineering Commons

\section{Recommended Citation}

B.M. Tymrak, M. Kreiger, J. M. Pearce, Mechanical properties of components fabricated with open-source 3-D printers under realistic environmental conditions, Materials \& Design, 58, pp. 242-246 (2014).

http://digitalcommons.mtu.edu/materials_fp/49

Follow this and additional works at: https://digitalcommons.mtu.edu/materials_fp

Part of the Materials Science and Engineering Commons 
B.M. Tymrak, M. Kreiger, J. M. Pearce, Mechanical properties of components fabricated with open-source 3-D printers under realistic environmental conditions, Materials \& Design, 58, pp. 242-246 (2014). http://dx.doi.org/10.1016/j.matdes.2014.02.038.

\title{
Mechanical Properties of Components Fabricated with Open-Source 3-D Printers Under Realistic Environmental Conditions
}

B. M. Tymrak, ${ }^{1}$ M. Kreiger, ${ }^{2}$ and J. M Pearce ${ }^{1,2,3, *}$

1.Department of Mechanical Engineering and Engineering Mechanics, Michigan Technological University, Houghton, MI, United States.

2. Department of Materials Science \& Engineering, Michigan Technological University, Houghton, MI, United States.

3.Department of Electrical \& Computer Engineering, Michigan Technological University, Houghton, MI, United States.

*corresponding author:pearce@mtu.edu

601 M\&M Building

1400 Townsend Drive

Houghton, MI 49931-1295

906-487-1466

\begin{abstract}
The recent development of the RepRap, an open-source self-replicating rapid prototyper, has made 3-D polymer-based printers readily available to the public at low costs $(<\$ 500)$. The resultant uptake of 3-D printing technology enables for the first time mass-scale distributed digital manufacturing. RepRap variants currently fabricate objects primarily from acrylonitrile butadiene styrene (ABS) and polylactic acid (PLA), which have melting temperatures low enough to use in melt extrusion outside of a dedicated facility, while high enough for prints to retain their shape at average use temperatures. In order for RepRap printed parts to be useful for engineering applications the mechanical properties of printed parts must be known. This study quantifies the basic tensile strength and elastic modulus of printed components using realistic environmental conditions for standard users of a selection of opensource 3-D printers. The results find average tensile strengths of 28.5 MPa for ABS and 56.6 MPa for PLA with average elastic moduli of $1807 \mathrm{MPA}$ for ABS and $3368 \mathrm{MPa}$ for PLA. It is clear from these results that parts printed from tuned, low-cost, open-source RepRap 3-D printers can be considered as mechanically functional in tensile applications as those from commercial vendors.
\end{abstract}

Keywords : 3-D printing; tensile strength; rapid prototyping; ABS; PLA

\section{Introduction}

Historically, expensive commercial rapid prototypers have enabled accurate fabrication of products or scale models, been useful as production and design tools, and the development of additive manufacturing (AM) for rapid prototyping in a number of technologies has been substantial [1-5]. Recently an open source (OS) model, the RepRap, has been developed that can be built for under $\$ 1000$ (now Prusa models can be made for about \$500), greatly expanding the potential user base of rapid prototypers. Between 2008 and 2011, it is estimated that the number of RepRaps in use had increased from 4 to 4500 [6], and can be assumed to have continued to increase in the last two years. In addition, other versions of at-home desktop 3-D printers are also selling rapidly. Makerbot, whose printers are derived from open-source RepRaps, for example, has sold over 13,000 3-D printers since 2009 [7]. The 
B.M. Tymrak, M. Kreiger, J. M. Pearce, Mechanical properties of components fabricated with open-source 3-D printers under realistic environmental conditions, Materials \& Design, 58, pp. 242-246 (2014). http://dx.doi.org/10.1016/j.matdes.2014.02.038.

resultant uptake of 3-D printing technology enables for the first time mass-scale environmentallybeneficial distributed digital manufacturing [8]. The RepRap was created by Adrian Bowyer and is supported and influenced by many contributors largely through the online wiki, which provides detailed assembly instructions for several variants of 3-D printers [6,9]. Thus following the OS model has created rapid technological evolution with the printers improving rapidly with time [10]. While OS models have limitations compared to commercial processes, they are capable of creating highly accurate parts with positioning accuracy of $0.1 \mathrm{~mm}$ [6]. RepRap variants currently fabricate objects primarily from acrylonitrile butadiene styrene (ABS) and polylactic acid (PLA), which have melting temperatures low enough to use in melt extrusion outside of a dedicated facility, while high enough for prints to retain their shape at average use temperatures. These machines are already used for art, toys, tools, household items (see Thingiverse an online repository of open 3-D printable designs) and to make high-value scientific instruments [11,12]. In addition, it has been proposed that RepRaps could be used for small-scale manufacturing or as an enabling tool for sustainable development [13]. In order to make RepRaps useful tools in this context and for standard engineering practice basic mechanical properties are necessary.

As RepRap 3D printers become more prevalent among home users they are being used to manufacture more diverse objects. This has included more load-bearing components that either replace items normally purchased or are uniquely designed for the specific needs of the user in terms of geometry and function. Both cases require the component to have the necessary strength properties to perform properly and safety. Most home users have no way of testing the strength of their parts and no extensive information is currently available about the mechanical properties of parts printed specifically on RepRaps.

To rectify this technical omission this study quantifies the basic tensile strength/stress, and elastic modulus of printed components using realistic environmental conditions for standard users of a selection of low-cost, open-source 3-D printers.

\section{Methods}

To determine the mechanical properties of 3-D printed parts and the variability in these properties when different user-controlled printing and slicing parameters are used, this investigation looked at the relationship between deposition pattern orientation and layer height to tensile strength, strain at tensile strength, and modulus. Table 1 shows the printing parameters used.

To gather a comprehensive data set covering a wide range of 3-D printers and their settings, a .STL file (as shown in Figure 1) of a tensile test specimen conforming to the ASTM D638 standard was created and distributed online for anyone to print and send to the researchers for testing [14,15]. An extra, unattached cylinder was added to the .STL file to aid in proper printing, but was not a part of the specimen.

A complete set of 10 specimens of each of the combinations of variables shown in Table 1 was printed on a variety of open-source 3-D printers including an original Mendel RepRap, a Prusa Mendel RepRap, a Lulzbot Prusa RepRap, and a custom MOST RepRap. The printers used (listed in Table 2) varied from each other with regard to mechanical design, including frame, stepper motors, and extruder head, as well as electronically with regard to firmware, with the open-source created Sprinter and 
B.M. Tymrak, M. Kreiger, J. M. Pearce, Mechanical properties of components fabricated with open-source 3-D printers under realistic environmental conditions, Materials \& Design, 58, pp. 242-246 (2014). http://dx.doi.org/10.1016/j.matdes.2014.02.038.

Marlin firmwares being the most commonly used. Different software was used for slicing the .STL files into machine readable g-code, which included Skeinforge, Slic3r, and Cura.

In order to determine realistic mechanical property values that RepRap users might encounter, the experiments diverged from the ASTM D638 standard because of uncontrollable specimen conditioning and geometry variability. To replicate realistic environmental conditions for distributed manufacturing, the environmental conditions during printing, storage, and shipping could not be controlled and no intentional specimen conditioning was performed.

While all specimens were created from the same .STL file, they were sliced and printed with different settings such as extruder temperature, based on which settings resulted in the best prints on each printer. Due to the nature of RepRaps and other user assembled 3-D printers being highly customizable, they can vary in construction and components resulting in different settings used in slicing and control software as well as in the firmware. One example of printer variability is how the temperature of the extruder is measured. Many different extruder models exist with most utilizing a thermistor for temperature measurement. Thermistor placement can vary substantially between models relative to the extruder heating element and nozzle. Thermistor calibration is also rarely, if ever, performed. This causes different printers to be set to different extruder temperatures to get high quality prints. Likewise, when two printers are set to the same temperature in software the actual extrusion temperature may be different. Observation has shown that a $5^{\circ} \mathrm{C}$ temperature change causes visible quality differences of a 3-D print, which is assumed to change the mechanical strength as well. Printer and slicing settings that were not specified for this study and subject to each printer's specific preferences were: extruder temperature, print bed temperature, nozzle diameter, cooling, print speed, and the number of extruded perimeters composing the outermost edge of a part.

The air gap between lines was not adjusted other than with the natural variability between printers. While parts can be created solid or hollow by adjusting the percent infill with RepRaps, with 100\% being completely solid, the exact air gap between extruded filament rasters cannot be specified. Therefore, while all specimens were printed with a setting of $100 \%$ infill, the actual positive or negative air gaps varies among printers due to printer differences. This affects the part as air gap has been shown to be an important contributing factor to tensile strength [16,17].

Testing was performed on an Instron 4468 load frame controlled using the Bluehill Software on a Windows PC. Load was measured with a $50 \mathrm{kN}$ load cell and strain measured using a 2 in gage length extensometer. Each test was conducted using a crosshead rate of $5 \mathrm{~mm} / \mathrm{min}$. Stress, strain, and modulus calculations were performed within the Bluehill Software. Each sample set tested consisted of ten specimens for a given group of printer settings.

Many specimens broke outside of the gage length due to assumed stress concentrations in the regions changing geometry as was also seen by [18]. Data was included in this study for specimens that broke out of the gage length, but displayed a distinct maximum stress before failure. For this reason conclusions could be made only for modulus and maximum strength, not specimen failure or elongation.

All specimens were printed in the x-y plane, which aligned the extruded filament with the direction of loading with the least number of layers. Orienting the build direction of the specimen in different planes changes the amount of extruded filament alignment with loading which can significantly affect tensile strength [19]. 
B.M. Tymrak, M. Kreiger, J. M. Pearce, Mechanical properties of components fabricated with open-source 3-D printers under realistic environmental conditions, Materials \& Design, 58, pp. 242-246 (2014). http://dx.doi.org/10.1016/j.matdes.2014.02.038.

\section{Results \& Discussion}

The data from testing showed variations between different specimen sets for both ABS and PLA, with some sets showing large internal differences. Table 3 shows the average property values for both PLA and ABS categorized by layer height and orientation.

For ABS, average values among both layer height and orientation showed no large discrepancies as all averages stayed with a reasonable range of the overall average. Specimens printed with a $0.2 \mathrm{~mm}$ layer height had the greatest tensile strength, while specimens at $0.4 \mathrm{~mm}$ layer height had the greatest elastic modulus. Between the $0^{\circ} / 90^{\circ}$ and $+45^{\circ} /-45^{\circ}$ orientations, $+45^{\circ} /-45^{\circ}$ was the strongest, while $0^{\circ} / 90^{\circ}$ had the greater elastic modulus.

The PLA specimens showed greater variability between parameters. For layer heights, tensile strength averages varied by $11.9 \mathrm{MPa}$, or $22 \%$, between $0.3 \mathrm{~mm}$ and $0.2 \mathrm{~mm}$ layer heights while elastic modulus varied by $194 \mathrm{MPa}$, or $6 \%$, between $0.4 \mathrm{~mm}$ and $0.2 \mathrm{~mm}$ layer height. Differences between values based on orientation were much smaller. The tensile strength for the $0.3 \mathrm{~mm}$ group was brought down by a set of 10 specimens with an average tensile strength of 35.4 MPa yet maintaining an average elastic modulus of $3342 \mathrm{MPa}$.

In general, most specimen sets (e.g. using the same printer and slicing conditions) grouped together, but there were some sets with a wide range, such as the $0.3 \mathrm{~mm}$ layer height $+45^{\circ} /-45^{\circ} \mathrm{ABS}$ set from Printer 1 spanning the right side of Figure 2.

Figures 2 and 3 show that the overall maximum values for both ABS and PLA were in specimens with a $0.2 \mathrm{~mm}$ layer height and $0^{\circ} / 90^{\circ}$ orientation. While this is more pronounced for ABS as the second highest set had a $0.2 \mathrm{~mm}$ layer height with a $+45^{\circ} / 45^{\circ}$ orientation, a potential indication of increased strength with layer height, both sets came from Printer 2, while both $0.2 \mathrm{~mm}$ layer height sets from Printer 1 had tensile strengths in the middle of the data for the $0^{\circ} / 90^{\circ}$ orientation set and at the bottom of the data for the $+45^{\circ} / 45^{\circ}$ orientation set.

On average, the mechanical property values of RepRap prints are higher than what has been found in similar studies of printed parts from commercial printers. ABS parts in a $0^{\circ}$ orientation have previously been found to have tensile strengths nearing $30 \mathrm{MPa}$ and elastic moduli around $1900 \mathrm{MPa}$ [20]. Other studies have shown tensile strengths varying between 10-18 MPa and moduli from 1000-1700MPa for various parameters $[17,19,21]$. One reason for higher values is most likely due to performing the tests at a higher strain rate than other studies. Higher strain rates have been shown to result in higher tensile strength values compared to a lower strain rate in printed parts [20]. However, it is clear from these results that parts printed from tuned, low-cost, open-source 3-D printers such as RepRaps can be considered as mechanically functional in tensile applications as those from commercial vendors. The number of perimeters a specimen has also been assumed to affect the strength. Perimeters are present on every layer and always align with the axis of loading in the gage length with one or two perimeters present on the specimens tested depending on user settings of each printer. Therefore, $+45^{\circ}$ $45^{\circ}$ orientations and $90^{\circ}$ layers were not complete as the perimeter had a $0^{\circ}$ orientation.

By observing the failure surfaces, many PLA specimens from the same printer with clear filament were found to have bonded during printing in such a way that the specimen appeared to be more like a homogeneous solid than a composition of individual extruded rasters. This is mostly likely due to a combination of high extruder temperature and filament that created significant thermal bonding 
B.M. Tymrak, M. Kreiger, J. M. Pearce, Mechanical properties of components fabricated with open-source 3-D printers under realistic environmental conditions, Materials \& Design, 58, pp. 242-246 (2014). http://dx.doi.org/10.1016/j.matdes.2014.02.038.

between both rasters and layers causing greater fusing than was seen in other specimens. These specimens also showed greater tensile strengths compared to specimens with distinct rasters, which indicates a potential method that RepRap users can utilize for components that demand higher strength. The type and quality of the polymer filament has also been observed to be an important factor in printing, whether in ABS or PLA. While printing specimens there were notable differences in extrusion characteristics when using different filaments, even when the only difference between filaments was color from the same vendor. Many different filament suppliers exist and identifiers about the exact filament composition are rarely provided to the end user at this time [22]. As low-cost 3-D printer use moves from the hobbyist to those making functional products for themselves or for others, filament vendors that provide both compositional, but also mechanical test data will be at a strategic advantage. While properties on average are similar, if not superior to commercial parts, care must be taken to assess part strength on a printer by printer basis for the following reasons: 1) Processing parameters, such as extrusion temperature, can have a significant effect on the structure and properties of printed parts. These parameters can change on a daily basis to get the best visual quality print therefore altering the mechanical properties. 2) The mechanical design of different printers and their components required different settings that can affect part strength. 3) Real parts loaded in various configurations can behave different than when in simple tension. 4) Filament quality and properties can vary substantially between suppliers (and possibly batches from the same supplier) and from environmental conditions resulting in changing properties.

Compared to conventional polymer processing, 3-D printing with RepRaps results in similar to weaker tensile strengths. Injection molded parts made from the same ABS polymer used for commercial printers has been shown to have a tensile strength of $26 \mathrm{MPa}$ [18] when tested at a lower strain rate. Other ABS polymers have shown to have tensile strengths from 34-43 $\mathrm{MPa}$ when injection molded [23-25]. For PLA, injection molded specimens have been seen to have tensile strengths from 30-63 MPa [26-28]. Therefore while RepRap printed parts are comparable in strength to commercial 3-D printers, for ABS RepRaps generally make parts weaker than conventional injection molding, but can have similar strengths to PLA injection molded parts.

With a wide range of variables not included in this study, such as extruder temperature, it is unclear as to whether the observed differences in mechanical properties were due to different layer heights and orientations or from other factors. Variability in part strength between different printers also suggests that unstandardized printer settings may play a more important role than uniformly controlled variables. As open-source 3-D printer use becomes widespread because of the economic advantages of distributed manufacturing for consumers [29], more scientific research is required to fully characterize the properties and abilities of printed parts. Focused work on identifying the relationship between print parameters and settings, especially extruder temperature, to part strength will help users create more functional components. Both the current wide selection of 3-D printer filaments available on the market, the burgeoning field of recycled waste plastic filament [30,31], as well as new materials commonly used in conventional manufacturing processes are becoming viable for 3-D printing and need to be mechanically characterized. Tests for flexural strength, impact strength, wear, thermal properties and electrical properties will provide valuable information about the functionality of 3-D printed parts as they start to be used in more diverse applications requiring higher performance. 
B.M. Tymrak, M. Kreiger, J. M. Pearce, Mechanical properties of components fabricated with open-source 3-D printers under realistic environmental conditions, Materials \& Design, 58, pp. 242-246 (2014). http://dx.doi.org/10.1016/j.matdes.2014.02.038.

Already RepRap technology has been developed for printing steel [32] and new tests will be needed to be developed to determine the mechanical properties of 3-D printed metal objects.

Finally, additional work is needed to address the impact of post-processing on the mechanical properties of the finished products. For example, to determine the impact of removing the support material from different geometric shapes with respect to interlayer direction. In addition, 3-D printed objects can be sanded and polished and painted to meet consumer preferences. Such post-processing steps can be chemical in nature as post-print chemical treatments have been developed for both ABS and PLA. ABS prints can be smoothed with acetone (nail polish remover) either by direct brush application or via a number of vapor treatments. PLA has been shown to be smoothed with a dip treatment in dichloromethane $\left(\mathrm{CH}_{2} \mathrm{Cl}_{2}, \mathrm{DCM}\right)$. Future work is needed to test these factors to assist a more broad application of distributed manufacturing with RepRaps.

\section{Conclusions}

The mechanical properties of ABS and PLA components made using various desktop open-source RepRap 3-D printers were characterized through standard tensile tests to determine tensile strength, strain at maximum strength and elastic modulus. The results show that the average tensile strength of RepRap printed parts are 28.5 MPa for ABS and 56.6 MPa for PLA with average elastic moduli of 1807 $\mathrm{MPa}$ for ABS and $3368 \mathrm{MPa}$ for PLA. These results indicate that the 3-D printed components from RepRaps are comparable in tensile strength and elastic modulus to the parts printed on commercial 3-D printing systems. While considerations must be made for the settings, tuning, and operation of each individual printer as well as the type, age, and quality of polymer filament used, functionally strong parts can be created with open-source 3-D printers within the bounds of their mechanical properties.

\section{Acknowledgments}

The authors would like to thank G. C. Anzalone and A. Perkins for helpful discussions and support. This material is based upon work supported by the National Science Foundation Graduate Research Fellowship under Grant No. DGE-1051031.

\section{References}

[1] Upcraft S, Fletcher R. The Rapid Prototyping Technologies. Assembly Automation 2003;23(4):31830. doi:10.1108/01445150310698634

[2] Gibson I, Rosen DW, Stucker B. Additive Manufacturing Technologies: Rapid Prototyping to Direct Digital Manufacturing. New York: Springer; 2010.

[3] Petrovic V, Gonzalez JVH, Ferrando OJ, Gordillo JD, Puchades JRB, Griñan LP. Additive layered manufacturing: sectors of industrial application shown through case studies. International Journal of Production Research 2011; 49(4);1061-79. doi10.1080/00207540903479786

[4] Gebhardt A, Schmidt FM, Hötter JS, Sokalla W, Sokalla P. Additive Manufacturing by selective laser melting the realizer desktop machine and its application for the dental industry. Physics Procedia 2010; 5: 543-49.doi:10.1016/j.phpro.2010.08.082

[5] Crane NB, Tuckerman J, Nielson GN. Self-assembly in additive manufacturing: opportunities and obstacles. Rapid Prototyping Journal 2011;17(3):211-17. doi:10.1108/13552541111124798 
B.M. Tymrak, M. Kreiger, J. M. Pearce, Mechanical properties of components fabricated with open-source 3-D printers under realistic environmental conditions, Materials \& Design, 58, pp. 242-246 (2014). http://dx.doi.org/10.1016/j.matdes.2014.02.038.

[6] Jones R, Haufe P, Sells E, Iravani P, Olliver V, Palmer C, Bowyer A. RepRap - the replicating rapid prototyper. Robotica 2011;29(1):177-91. doi:10.1017/S026357471000069X

[7] Makerbot FAQ. http://www.makerbot.com/faq/

[8] Kreiger M, Pearce JM. Environmental Impacts of Distributed Manufacturing from 3-D Printing of Polymer Components and Products. MRS Online Proceedings Library 2013; 1492.

doi:10.1557/opl.2013.319

[9] Build a RepRap. RepRapWiki. http://reprap.org/wiki/Build A RepRap

[10] Chulilla JL. The Cambrian Explosion of Popular 3D Printing. International Journal of Interactive Multimedia and Artificial Intelligence 2011;1(4):30-2. doi:10.9781/ijimai.2011.145

[11] Pearce JM. Building Research Equipment with Free, Open-Source Hardware. Science 2012;337(6100):1303-4. doi:0.1126/science.1228183

[12] Pearce JM. Open-Source Lab How to Build Your Own Hardware and Reduce Research Costs, Elsevier, 2014.

[13] Pearce JM, Morris Blair C, Laciak KJ, Andrews R, Nosrat A, Zelenika-Zovko I. 3-D Printing of Open Source Appropriate Technologies for Self-Directed Sustainable Development. Journal of Sustainable Development 2010;3(4):17-29.

[14] ASTM Standard D638-10, 2010. Standard Test Methods for Tensile Properties of Plastics. ASTM International, West Conshohocken, PA, 2010.

[15] Revised ASTM Tensile Test Specimen. http://www.thingiverse.com/thing:28987.

[16] Rodriguez JF, Thomas JP, Renard JE. Design of Fused-Deposition ABS Components for Stiffness and Strength. Journal of Mechanical Design 2003;125(3):545-51. doi:10.1115/1.1582499

[17] Li L, Sun Q, Bellehumeur C, Gu P. Composite Modeling and Analysis for Fabrication of FDM Prototypes with Locally Controlled Properties. Journal of Manufacturing Processes 2002;4(2):129-41.

[18] Ahn SH, Montero M, Odell D, Roundy S, Wright PK. Anisotropic material properties of fused deposition modeling ABS. Rapid Prototyping Journal 2002;8(4):248-57. doi: $10.1108 / 13552540210441166$

[19] Bellini A, Güçeri S. Mechanical characterization of parts fabricated using fused deposition modeling. Rapid Prototyping Journal 2003;9(4):252-64. doi:10.1108/13552540310489631

[20] Rodriguez JF, Thomas JP, Renaud JE. Mechanical behavior of acrylonitrile butadiene styrene (ABS) fused deposition materials. Experimental investigation. Rapid Prototyping Journal 2001;7(3): 148-58. doi:10.1108/13552540110395547

[21] Sood AK, Ohdar RK, Mahapatra SS. Parametric appraisal of mechanical property of fused deposition modelling processed parts. Materials \& Design 2010;31(1):287-95. doi:10.1016/j.matdes.2009.06.016

[22] 3ders.org. Price compare 3d printing materials. http://www.3ders.org/pricecompare/

[23] Ozcelik B, Ozbay A, Demirbas E. Influence of injection parameters and mold materials on mechanical properties in plastic injection molding. International Communications in Heat and Mass Transfer 2010;37;1359-65. doi:10.1016/j.icheatmasstransfer.2010.07.001

[24] Fu SY, Lauke B. Characterization of tensile behavior of hybrid short glass fibre/calcite particle/ABS composites. Composites Part A 1990; 29A:575-83. 
B.M. Tymrak, M. Kreiger, J. M. Pearce, Mechanical properties of components fabricated with open-source 3-D printers under realistic environmental conditions, Materials \& Design, 58, pp. 242-246 (2014). http://dx.doi.org/10.1016/j.matdes.2014.02.038.

[25] Scaffaro R, Botta L, Di Benedetto G. Physical properties of virgin-recycled ABS blends: Effect of post-consumer content and of reprocessing cycles. European Polymer Journal 2012;48:637-48. doi:10.1016/j.eurpolymj.2011.12.018

[26] Graupner N, Herrmann AS, Müssig J. Natural and man-made cellulose fibre-reinforced poly(lactic acid) (PLA) composites: An overview about mechanical characteristics and application areas. Composites Part A 2009; 40:810-21. doi:10.1016/j.compositesa.2009.04.003

[27] Oksman K, Skrifvars M, Selin JF. Natural fibers as reinforcement in polylactic acid (PLA) composites. Composites Science and Technology 2003;63:1317-24. doi:10.1016/S02663538(03)00103-9

[28] Bledzki AK, Jaszkiewicz A, Scherzer D. Mechanical properties of PLA composites with manmade cellulose and abaca fibres. Composites Part A 2009; 40:404-12. doi:10.1016/j.compositesa.2009.01.002

[29] Wittbrodt BT, Glover AG, Laureto J, Anzalone GC, Oppliger D, Irwin JL, Pearce JM. Life-cycle economic analysis of distributed manufacturing with open-source 3-D printers. Mechatronics 2013; 23(6): 713-726. doi:10.1016/j.mechatronics.2013.06.002

[30] Baechler C, DeVuono M, Pearce JM. Distributed Recycling of Waste Polymer into RepRap Feedstock. Rapid Prototyping Journal 2013; 19(2):118-125. doi:10.1108/13552541311302978

[31] Kreiger M, Anzalone GC, Mulder ML, Glover A, Pearce JM. Distributed Recycling of Post-Consumer Plastic Waste in Rural Areas. MRS Online Proceedings Library 2013; 1492, mrsf121492-g04-06.

[32] Anzalone GC, Zhang C, Wijnen B, Paul G. Sanders PG, Pearce JM. Low-Cost Open-Source 3-D Metal Printing. IEEE Access (in press). doi:10.1109/ACCESS.2013.2293018 
B.M. Tymrak, M. Kreiger, J. M. Pearce, Mechanical properties of components fabricated with open-source 3-D printers under realistic environmental conditions, Materials \& Design, 58, pp. 242-246 (2014). http://dx.doi.org/10.1016/j.matdes.2014.02.038.

\section{Figure Captions}

Figure 1. a) Rendering of the shared .STL filed of the ASTM D638 tensile standard [15] and b) digital photograph of a specimen in load frame.
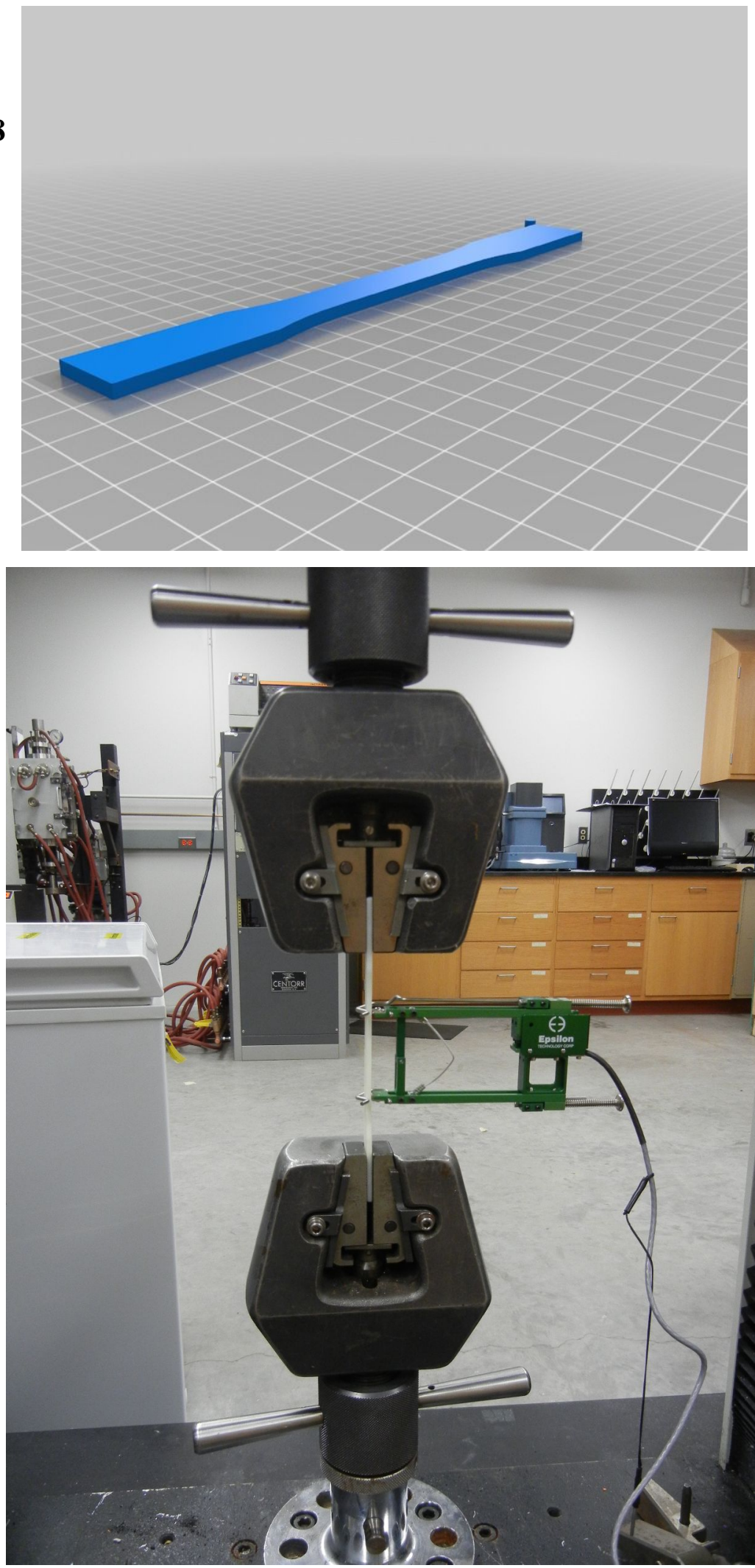
B.M. Tymrak, M. Kreiger, J. M. Pearce, Mechanical properties of components fabricated with open-source 3-D printers under realistic environmental conditions, Materials \& Design, 58, pp. 242-246 (2014). http://dx.doi.org/10.1016/j.matdes.2014.02.038.

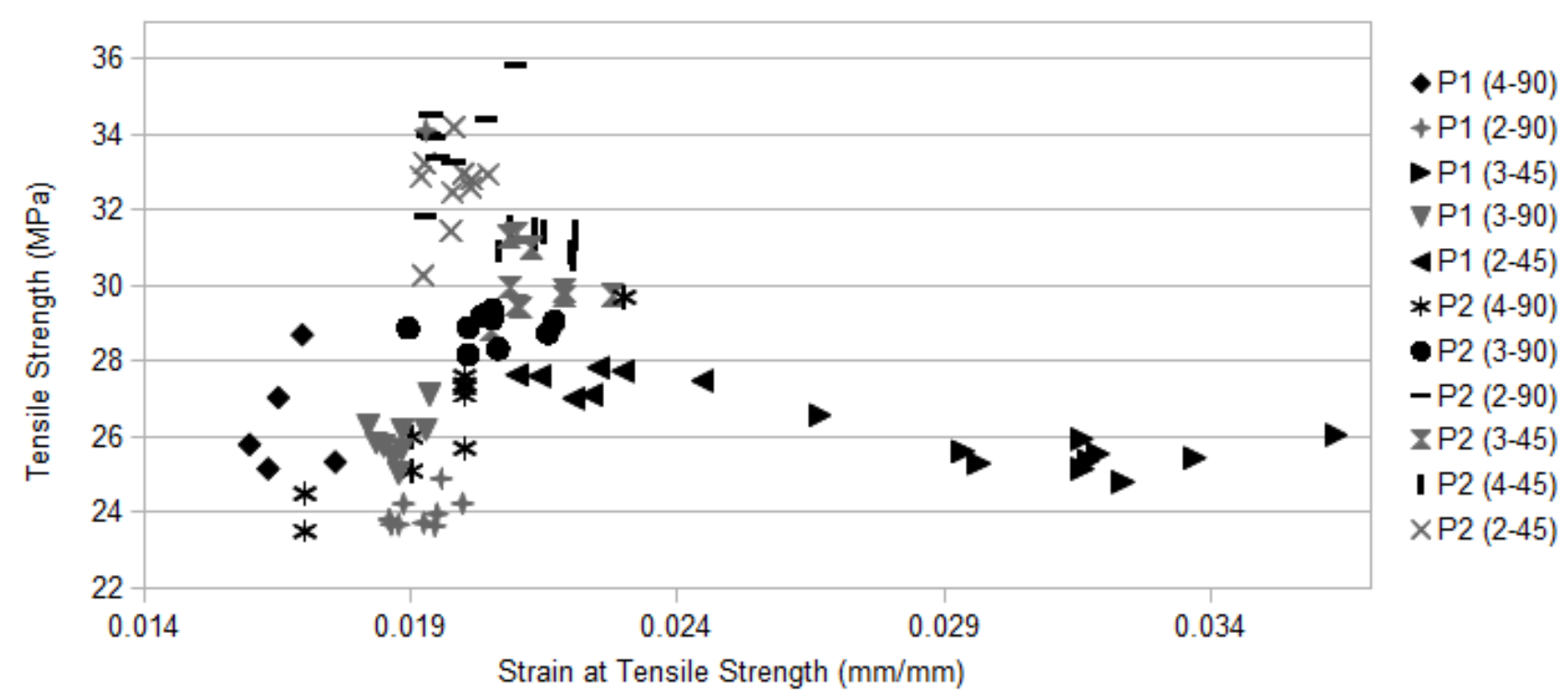

Figure 2. Tensile Strength vs. Strain at Tensile Strength for ABS printed specimens. Legend: Printer name (layer height-orientation).

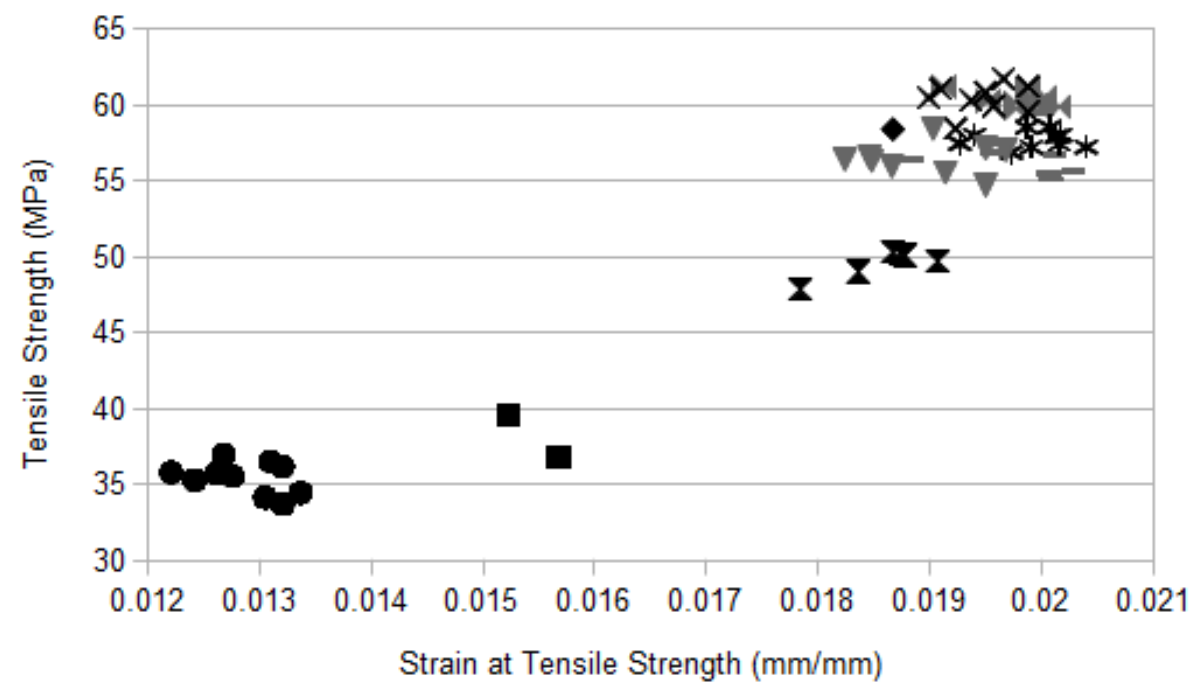

Figure 3. Tensile Strength vs. Strain at Tensile Strength for - P1 (4-90) PLA printed specimens. VP1 (3-90) Legend: Printer name $\times \mathrm{P} 1(2-90)$ *P1 (4-45) $-\mathrm{P} 1(3-45)$ (layer heightMP1 (2-45) x $\mathrm{P} 2$ (4-90) - $P 3$ (3-90) - P4 (3-45) 